\title{
STABILITY ANALYSIS OF STRIP FOOTING ON REINFORCED SAND SLOPES
}

\author{
RAFi' M. Sulaiman AL-NE'Aimi \\ College of Engineering, University of Duhok, Kurdistan Region-Iraq
}

(Received: January 3, 2018; Accepted for Publication: November 26, 2018)

\begin{abstract}
This paper presents a total of 55 runs using Slide v6 software computer program to study the stability of axially loaded strip footing located near the crest of sand slope model without or with geogrid reinforcement. The model represents an application of reinforced earth technique for foundations located on sloped embankments that behave as supports for retaining walls or bridge abutments. The parameters investigated were slope inclination $\beta$, footing distance from edge of the slope $b$, length and number of geogrid layers $L_{r}$ and $N$, type of geogrid reinforcement in terms of its structure, polymer type, yield elongation $\%$ and strength, vertical spacing between geogrid layers $\Delta v$ and depth of embedment of footing $D_{f}$.

The limit equilibrium analysis results show that (i) Slide v6 software program is efficient to study unreinforced or reinforced slope stability problems both for research or design purposes, (ii) Use of geogrid reinforcement would increase both slope stability and safety. The major benefit was obtained when reinforcing layers number $N$ equal to four each of length $10 B$ where (B = width of footing), (iii) SS30 geogrid is more effective for reinforcing slopes than TT045 type due to its higher pullout strength and other properties such as structure, shape, rib profile and grid junction tensile strength that affecting soil/grid interlock, and (iv) the optimum depth of the upper most layer of reinforcement was $u=0.5 B$ whereas the optimum vertical spacing of the reinforcement layers $\Delta v$ lies in the range of $0.50 \mathrm{~B}-0.75 \mathrm{~B}$.
\end{abstract}

KEYWORDS: Reinforced sand slope, Strip footing, geogrid reinforcement, Limit- equilibrium analysis, Slide v6 program.

\section{INTRODUCTION}

F oundations were sometimes built on or near slopes for such buildings or roads in hilly regions and sloped embankments that used as supports for bridge abutments. The position of foundation relative to the edge of fill sloped embankment has evident effects not only on economic efficiency of the overall bridge structure design but also on its safety due to slope stability failure, Choudhary et al. (2010).

Several improvement techniques have been proposed in literature to improve the load carrying capacity of such foundations located on fill sloped embankments. Incorporation of deep deposits of granular fill materials, incorporation of piles, using geosynthetics or geogrids as reinforcements, etc... are common techniques. Lee and Manjunath (2000) compared three different types of geosynthetic reinforcement using single layer. The maximum improvement in bearing capacity, was obtained when the geogrid layer was placed at $0.5 \mathrm{~B}$ depth, where B is the width of footing, Whereas, Yoo (2001) 's study on laboratory slope model of strip footing reinforced by multiple layers of geogrid reinforcement showed that to gain maximum improvement in bearing capacity, the first geogrid layer should be laid at 1.0B depth. Laman and Yildiz (2003) reported that geogrids generally mobilize higher bond stress with soil than geotextiles. This was mainly due to the combined effect of soil-reinforcement interaction results from frictional, interlocking and adhesion properties of reinforcement with soil. Some researchers such as; Lee and Manjunath (2000), Gill et al. (2011), and Keskin and Laman (2014) studied strip footings on geogrid-reinforced sand slope experimentally and numerically using PLAXIS software. Their results showed that bearing capacity improvement depends on slope angle, relative density of sand, and the geogrid reinforcement tensile strength.

This work presents a modelling study of loaded strip footing located near crest of unreinforced and geogrid-reinforced sand slope using Slide v6 software program. The model testing program involves several parameters such as; slope inclination $\beta$, footing distance from edge of the slope $b$, length and number of geogrid layers $L_{r}$ 
and $N$, type of geogrid reinforcement, vertical spacing between geogrid layers $\Delta v$ and depth of embedment of footing $D_{f}$.

\section{REINFORCED SOIL CONCEPT}

The concept of reinforced soil was explained through the following mechanisms:

\subsection{Stress Transfer Mechanisms}

(a) Friction: The soil particles, in direct contact with the reinforcement such as metal strips, geotextiles and geogrid layers, tend to slide over reinforcement under the effect of normal load. The sliding is prevented by frictional resistance between the soil particles and the reinforcement surfaces. Then, this resistance generates tensile forces along the reinforcement as shown in Fig.(1a), Elias et al. (2001).

(b) Passive Resistance: It is developed as bearing stresses on "transverse" surfaces of geogrids, bar mats, and wire mesh reinforcements normal to the direction of soil-reinforcement relative movement as shown in Fig.(1b). The contribution of each transfer mechanism for a particular reinforcement will depend on the surface skin friction, normal effective stress, grid opening dimensions, thickness of transverse members, and elongation characteristics of the reinforcement, Elias et al. (2001).
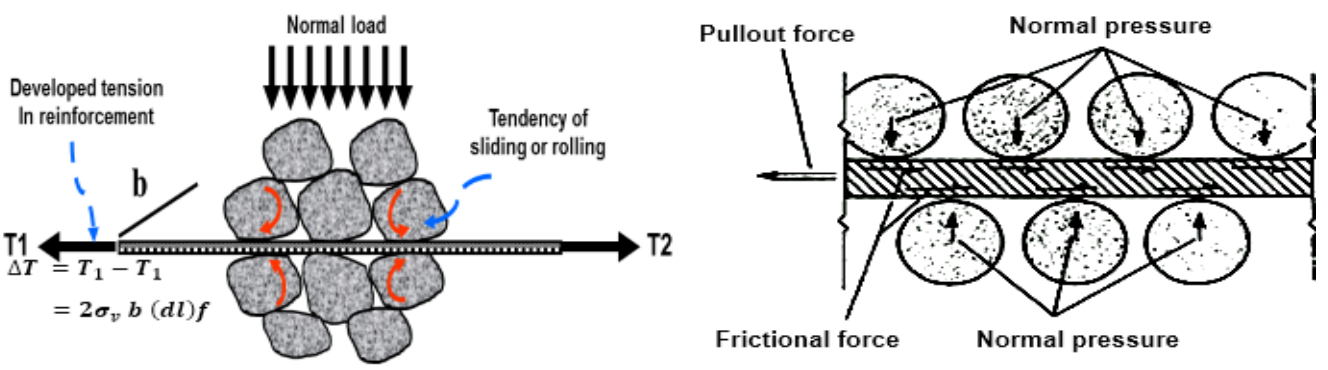

(a) Frictional stress transfer between soil and reinforcement surfaces.
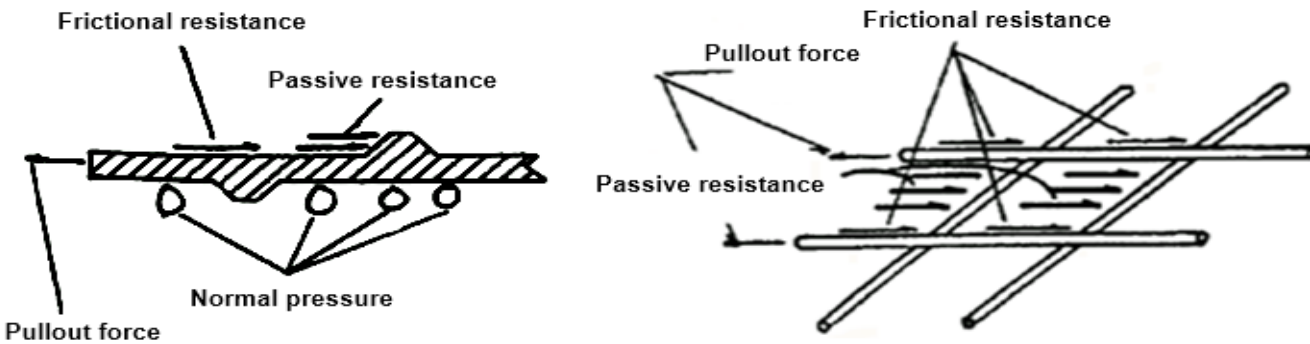

(b) Soil passive resistance on reinforcement surfaces.

Fig. (1): Stress transfer mechanisms for soil reinforcement (after Elias et al. 2001).

\subsection{Reinforcement Mechanisms}

Generally, reinforcements could withstand the soil deformations in two ways, Elias et al. (2001):

(a) Tension: All "longitudinal" reinforcing elements can resist tensile stresses developed which in turn act to restrict the soil movements (especially in horizontal direction that almost gives lateral strain close to zero) as shown in Fig.(2), and impart additional shear strength in composite soil/reinforcement system than soil mass alone.

(b) Shear Stress and Bending: These stresses could be resisted by "Transverse" reinforcing elements that have some rigidity. 

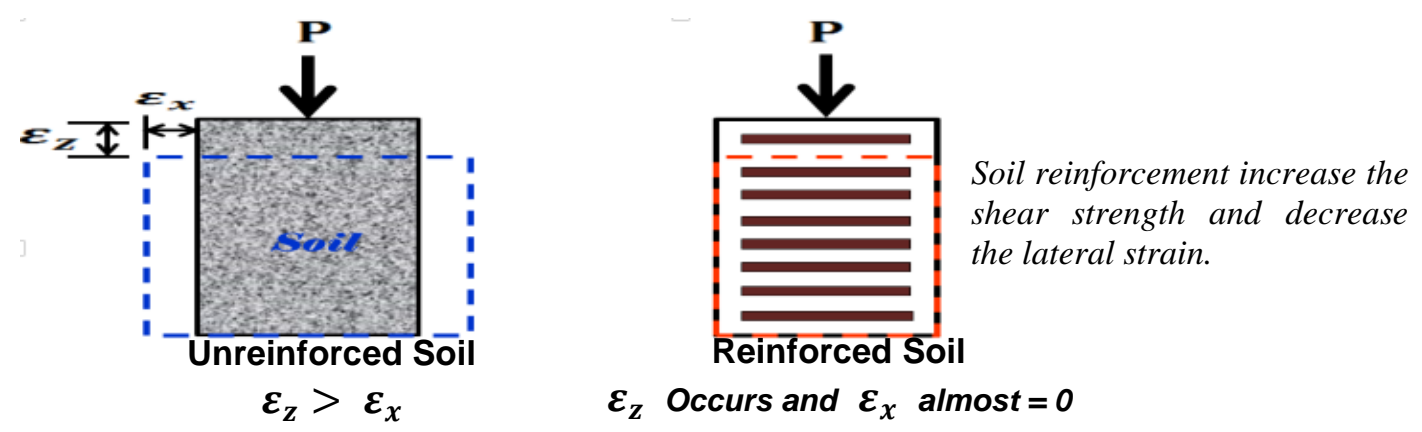

Fig. (2): Lateral strain for unreinforced and reinforced soil (after Elias et al. 2001).

\section{MODES OF FAILURE OF REINFORCED SLOPES}

All requirements for design of reinforced slopes are similar to those of unreinforced ones. In general, for all possible failure modes, the safety factor must be adequate for both short-term and long-term conditions. As illustrated in Fig.(3), three possible failure modes in reinforced slopes could be observed, Strata Systems (2010):-

1. External Failure Modes, these modes involve sliding, overturning, bearing capacity, and deep seated failures. It occurs when the failure plane passes behind and underneath the reinforced soil mass in case of poor backfill material and/ or soft foundation soil strata of low strength.

2. Internal Failure Modes, these modes include tensile and pullout failures. It occurs when the failure plane passes through the reinforcing elements.

3. Compound Failure Modes, these modes occur when the failure plane passes behind and through the reinforced soil mass.

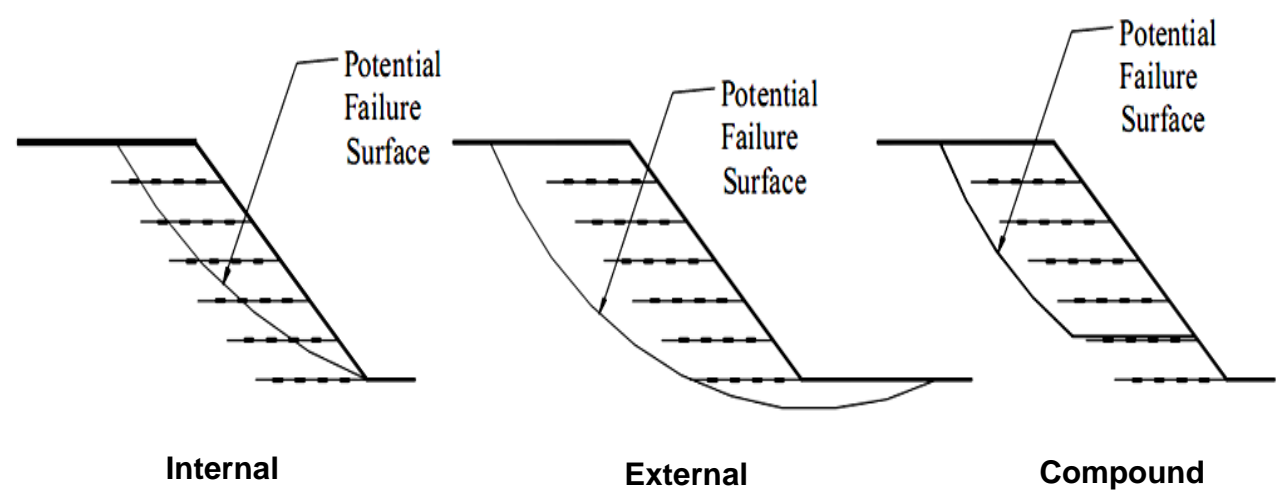

Fig. (3): Failure modes of reinforced slopes (after Strata Systems, 2010).

\section{SAFETY FACTOR OF SOIL SLOPES}

\subsection{Unreinforced Slope}

The safety factor $\mathrm{FS}_{\mathrm{u}}$ of unreinforced slope was expressed as (Strata Systems, 2010):

$F S_{u}=\frac{1}{\sum W \sin \psi} \sum\left[\frac{\left\{c^{\prime} b+W\left(1-r_{u}\right) \tan \phi\right\} \sec \psi}{\frac{1+\tan \psi \tan \phi^{\prime}}{F S_{u}}}\right]$

where, the summation signs in Eq.(1) are with respect to the vertical slices shown in Fig.(4), and
$\mathrm{w}=$ total weight of slice based on bulk unit weight of soil plus surcharge loading if present,
$\mathrm{q}=$ uniformly distributed surcharge acting at crest of slope, 
$\mathrm{b}=$ the horizontal width of the slice,

$\psi=$ the angle formed by the tangent to the midpoint of the slice and the horizontal

$\mathrm{c}^{\prime}=$ soil cohesion at base of slice,

$\emptyset^{\prime}=$ peak soil friction angle at base of slice,

$\mathrm{r}_{\mathrm{u}}=$ pore water pressure coefficient determined using the approach shown in Fig.(4b) and equal to zero $\left(r_{u}=0\right)$ for any slice that does not intersect the groundwater table.

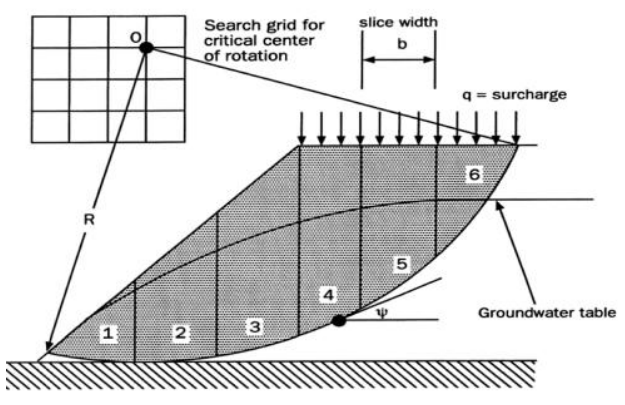

(a) Method of Slices

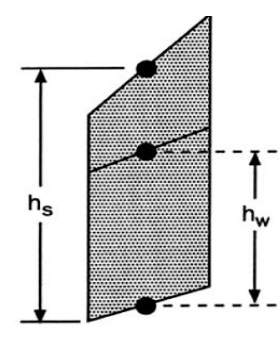

$r_{u}=\frac{u}{W / b} \approx \frac{h_{w} / w}{W / b}$

$u=$ porewater pressure at base

of slice

$\gamma_{w}=$ unit weight of water

(b) Pore water pressure coefficient.

Fig. (4): Circular Slip Analysis for Unreinforced Slope (after Mirafi, 1998).

\subsection{Reinforced Slope}

The safety factor $\mathrm{FS}_{\mathrm{r}}$ of reinforced slope was expressed as (Strata Systems, 2010):

$$
F S_{r}=F S_{u}+\frac{\text { Resisting movment due to reinforcement }}{\text { Driving moment }}
$$

or

$$
F S_{r}=F S_{u}+\left(\frac{\sum T_{i} R_{T i} / \cos \psi_{1}}{M_{D}}\right)
$$

where, the summation term is related to reinforcement layers and tangent slopes of the circular slip surface at points of intersections with each reinforcement layer $\boldsymbol{i}$ as shown in Fig.(5), and $M_{D}$ is the driving moment of all forces causing failure.

The magnitude of the tensile force $\boldsymbol{T}_{\boldsymbol{i}}$ used for each layer in the summation term in Eq.(3) must be the lesser of:
1. The working tensile load level below which the reinforcement remains intact and does not undergo excessive straining.

2. The pullout capacity of the embedded length of the reinforcement beyond the slip circle (i.e., $\begin{array}{lllll}\text { length } & \boldsymbol{l}_{\boldsymbol{a}} & \text { shown in Fig.(5)) }\end{array}$

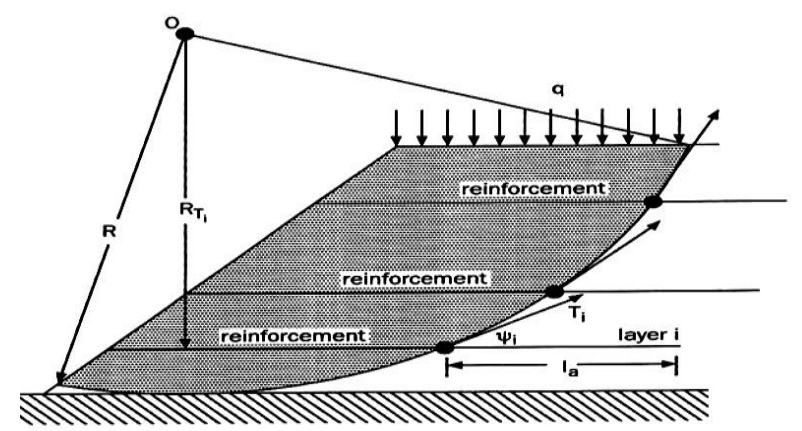

Fig. (5): Circular slip analysis for reinforced slope (after Mirafi, 1998). 


\section{Modelling of Slope Using Slide Software}

\subsection{Material Properties Used 5.1.1 Backfill Sand}

In this study, the dry medium to coarse sand used by Alamshahi and Hataf (2009) was selected as a backfill material of the model slope. The grain size distribution curve of the sand is shown in Fig.(6) and its properties is presented in Table (1).

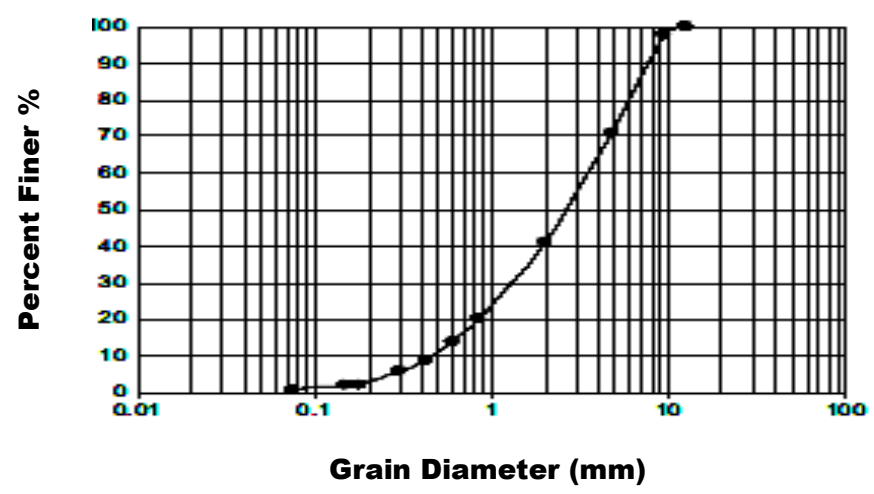

Fig. (6): Grain size distribution of the sand (after Alamshahi and Hataf, 2009).

Table (1): Properties of sand used in present stability analysis (after Alamshahi and Hataf, 2009).

\begin{tabular}{ll}
\hline Parameter & Value \\
\hline Cohesion, c $(\mathrm{kPa})$ & 0.0 \\
Residual angle of internal friction $\left(\varnothing^{o}\right)$ & 38 \\
Angle of dilatancy $\left(\psi^{o}\right)$ & 5 \\
Dry unit weight, $\gamma\left(\mathrm{kN} / \mathrm{m}^{3}\right)$ & 16.9 \\
Modulus of elasticity, $\mathrm{E}\left(\mathrm{kN} / \mathrm{m}^{2}\right)$ & \\
Poisson's ratio, $v$ & 20000 \\
Maximum dry density $\left(\mathrm{kN} / \mathrm{m}^{3}\right)$ & 0.3 \\
Minimum dry density $\left(\mathrm{kN} / \mathrm{m}^{3}\right)$ & \\
Relative density, $\operatorname{Dr}(\%)$ & 18.73 \\
& 13.92 \\
& 70 \\
\hline
\end{tabular}

\subsubsection{Footing}

In this study, a strip footing of $80 \mathrm{~mm}$ width and $600 \mathrm{~mm}$ length was used. It was made out of an aluminum alloy with a thickness of $40 \mathrm{~mm}$ to resist bending moment under the applied load.

\subsubsection{Geogrid Reinforcements}

Two types of plastic geogrid materials with different stiffness and strength values were used as

\begin{tabular}{ll}
\hline Parameter & Value \\
\hline $\mathrm{D}_{10}(\mathrm{~mm})$ & 0.45 \\
$\mathrm{D}_{30}(\mathrm{~mm})$ & 3.5 \\
$\mathrm{D}_{60}(\mathrm{~mm})$ & 1.5 \\
Coefficient of uniformity, $\mathrm{C}_{\mathrm{u}}$ & 7.78 \\
Coefficient of curvature, $\mathrm{C}_{\mathrm{c}}$ & 1.42 \\
Classification (USCS) & $\mathrm{SW}$ \\
& \\
\hline
\end{tabular}

a reinforcing material. Table (2) shows the index properties of the used materials based on tensile testing carried out in accordance with the American Society for Testing and Materials test method D4595-96 (ASTM 1996). The geometry of these products is illustrated in Fig.(7). 
Table (2): Index properties of used geogrid reinforcements (after ASTM 1996).

\begin{tabular}{|c|c|c|}
\hline Property & TENAX TT 045 SAMP & Tensar SS30 \\
\hline $\begin{array}{l}\text { Structure } \\
\text { Aperture size }(\mathrm{mm} \times \mathrm{mm}) \\
\text { Weight }\left(\mathrm{gm} / \mathrm{m}^{2}\right) \\
\text { Polymer type } \\
\text { Tensile strength at } 2 \% \text { strain }(\mathrm{kN} / \mathrm{m}) \\
\text { Tensile strength at } 5 \% \text { strain }(\mathrm{kN} / \mathrm{m}) \\
\text { Peak tensile strength }(\mathrm{kN} / \mathrm{m}) \\
\text { Yield point elongation }(\%) \\
\text { Pullout strength @ } \mathrm{Dr}=70 \%: \\
\text { - Adhesion }(\mathrm{kPa})^{* *} \\
\text { - Friction Angle (Degree) } \\
{ }^{* *}\end{array}$ & $\begin{array}{l}\text { Uniaxillay oriented geogrids } \\
(13 / 20) \times 220 \\
300 \\
\text { HDPE * } \\
11 \\
25 \\
45 \\
11.5 \\
\\
2.4 \\
15.7\end{array}$ & $\begin{array}{l}\text { Bi-axially oriented grids } \\
39 \times 39 \\
330 \\
\text { PP * } \\
10.5 \\
21 \\
30 \\
13 \\
13.1 \\
29\end{array}$ \\
\hline
\end{tabular}

* HDPE, uniaxial high-density polyethylene geogrid; PP, biaxial polypropylene geogrid.

** (from Shi and Wang, 2013).

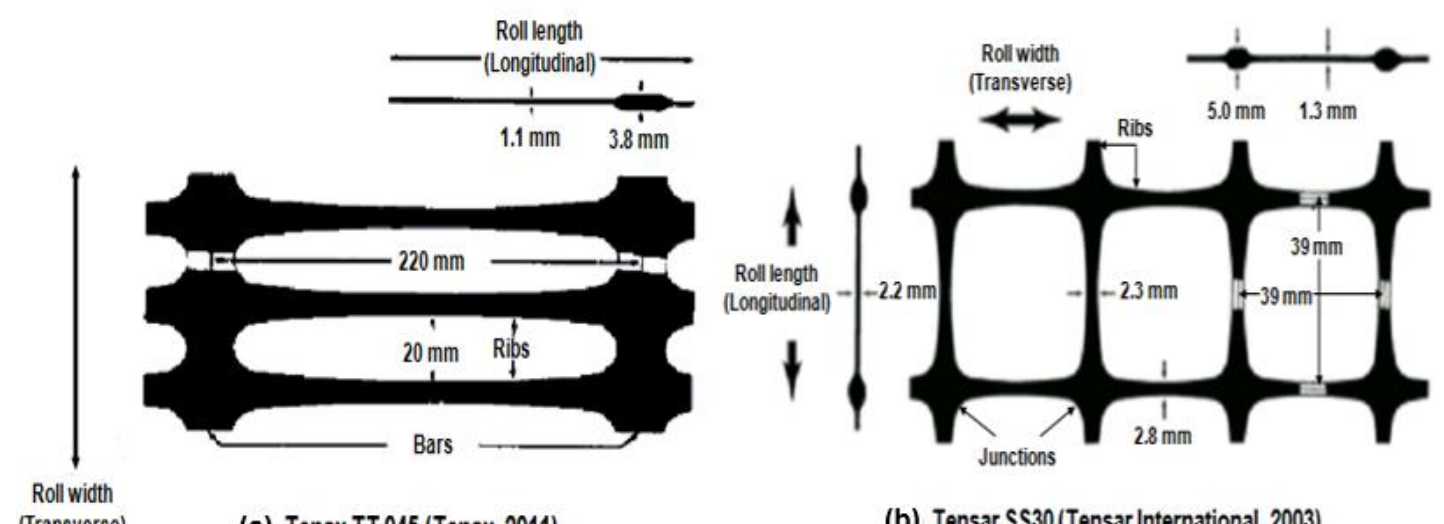

(Transverse) $\quad$ (a) Tenax TT 045 (Tenax, 2011)

(b) Tensar SS30 (Tensar International, 2003)

FIGURE (7): Geometry of used geogrid reinforcements.

5.2 Model Slope Geometry and Testing Program

Referring to the model slope geometry shown in Fig.(8), a total of 55 runs as presented in Table (3) were conducted in order to study the stability of axially loaded plane strain footing resting on unreinforced and reinforced sand model slope geometry using the 2D Slide v6 slope stability software

program. 


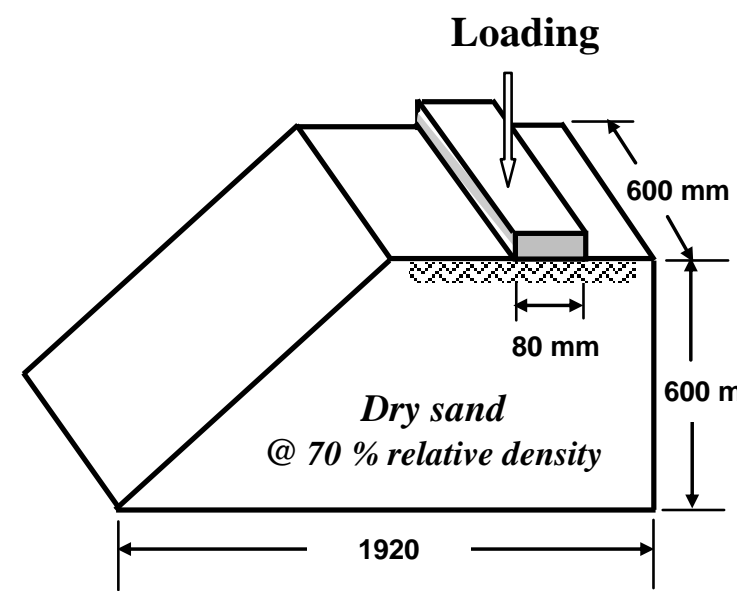

(a) Slope model size

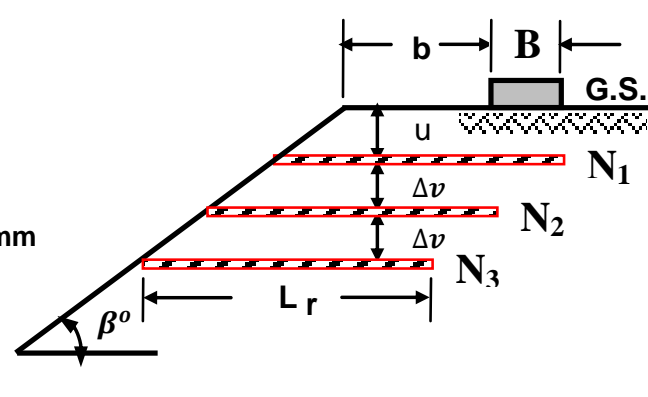

$\mathrm{N}_{\mathrm{i}}=$ No. of geogrid reinforcement layers.

(b) Studied parameters.

Fig (8): Schematic view of model slope geometry.

Table (3): Model Testing Program for sand slope analysis.

\begin{tabular}{|c|c|c|c|c|c|c|c|c|c|}
\hline $\begin{array}{c}\text { Series } \\
\text { No. }\end{array}$ & $\begin{array}{l}\text { Test } \\
\text { No. }\end{array}$ & $\bar{\beta}$ & $b$ & $\mathrm{~N}$ & $L_{r}$ & $\begin{array}{c}\text { Type } \\
\text { of } \\
\text { geogrid }\end{array}$ & $\Delta v$ & $\overline{D_{f}}$ & $\begin{array}{c}\text { Tests conditions } \\
\text { and } \\
\text { Studied Parameters }\end{array}$ \\
\hline 1 & $1-12$ & $\begin{array}{l}30^{\circ} \\
35^{\circ} \\
40^{\circ}\end{array}$ & $\begin{array}{l}1 \mathrm{~B} \\
2 \mathrm{~B} \\
3 \mathrm{~B} \\
4 \mathrm{~B}\end{array}$ & 0 & ----- & ------- & ----- & 0 & $\begin{array}{l}\text { Applied line load = } 50 \mathrm{kN} / \mathrm{m} \\
\text { Slope angle, } \beta=? \\
\text { Footing distance from edge of } \\
\text { slope, } b=?\end{array}$ \\
\hline 2 & $13-20$ & $35^{\circ}$ & $\begin{array}{l}1 B \\
2 B \\
3 B \\
4 B\end{array}$ & 4 & $\begin{array}{c}7 \mathrm{~B} \\
10 \mathrm{~B}\end{array}$ & SS30 & $0.5 B$ & 0 & $\begin{array}{l}\text { Applied line load }=50 \mathrm{kN} / \mathrm{m} \\
\text { Depth of the first layer of } \\
\text { geogrid reinforcement, } \mathrm{u}=0.5 \mathrm{~B} \\
\text { Length of geogrid, } L_{r}=?\end{array}$ \\
\hline 3 & $21-40$ & $35^{\circ}$ & $\begin{array}{l}1 \mathrm{~B} \\
2 \mathrm{~B} \\
3 \mathrm{~B} \\
4 \mathrm{~B}\end{array}$ & $\begin{array}{l}1 \\
2 \\
3 \\
4 \\
5\end{array}$ & 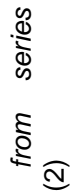 & SS30 & $0.5 \mathrm{~B}$ & 0 & $\begin{array}{l}\text { Applied line load }=50 \mathrm{kN} / \mathrm{m} \\
\mathrm{u}=0.5 \mathrm{~B} \\
\text { Number of reinforcing layers, } \mathrm{N} \\
=?\end{array}$ \\
\hline 4 & $41-48$ & $35^{\circ}$ & $\begin{array}{l}1 \mathrm{~B} \\
2 \mathrm{~B} \\
3 \mathrm{~B} \\
4 \mathrm{~B}\end{array}$ & 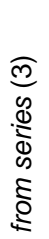 & 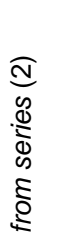 & $\begin{array}{l}\text { TT045 } \\
\text { SS30 }\end{array}$ & $0.5 B$ & 0 & $\begin{array}{l}\text { Applied line load }=50 \mathrm{kN} / \mathrm{m} \\
\mathrm{u}=0.5 \mathrm{~B} \\
\text { Type of reinforcement }=?\end{array}$ \\
\hline
\end{tabular}


Table (3): Cont'd.

\begin{tabular}{|c|c|c|c|c|c|c|c|c|c|}
\hline $\begin{array}{c}\text { Series } \\
\text { No. }\end{array}$ & $\begin{array}{l}\text { Test } \\
\text { No. }\end{array}$ & $\beta$ & b & $\mathrm{N}$ & $L_{r}$ & $\begin{array}{l}\text { Type } \\
\text { of } \\
\text { geogrid }\end{array}$ & $\Delta v$ & $D_{f}$ & $\begin{array}{c}\text { Tests conditions } \\
\text { and } \\
\text { Studied Parameters }\end{array}$ \\
\hline 5 & $49-53$ & $35^{\circ}$ & $4 B$ & 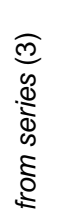 & 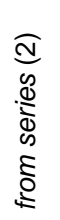 & 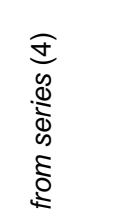 & $\begin{array}{l}0.25 \mathrm{~B} \\
0.50 \mathrm{~B} \\
0.75 \mathrm{~B} \\
1.00 \mathrm{~B} \\
1.50 \mathrm{~B}\end{array}$ & 0 & $\begin{array}{l}\text { Applied line load }=50 \mathrm{kN} / \mathrm{m} \\
\mathrm{U}=0.5 \mathrm{~B} \\
\text { Vertical spacing between } \\
\text { reinforcement layers, } \Delta v=?\end{array}$ \\
\hline 6 & $54-55$ & $35^{\circ}$ & $4 \mathrm{~B}$ & 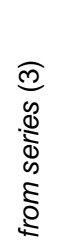 & 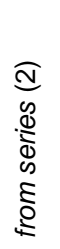 & 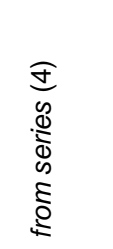 & 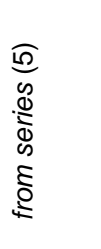 & $\begin{array}{c}0 \\
0.5 \mathrm{~B} \\
1.0 \mathrm{~B}\end{array}$ & $\begin{array}{l}\text { Applied line load }=50 \mathrm{kN} / \mathrm{m} \\
\mathrm{u}=0.5 \mathrm{~B} \\
\text { Depth of footing, } D_{f}=?\end{array}$ \\
\hline
\end{tabular}

$\mathbf{B}=$ Width of strip footing.

\section{SIMULATION ANALYSIS RESULTS}

\subsection{Effect of slope inclination angle and footing location from slope edge}

Table (4) shows the test results of series No.1 described as safety factors obtained from application of Slide v6 software using five different analysis methods. The table shows that for any slope angle $\beta$ considered, the safety factor (S.F.) increases as the footing distance away from edge of slope $b / B$ increases. Whereas for the same $b / B$, as the slope angle $\beta$ increases, the S.F. is significantly decreases. The same trend was noticed in all used methods of analysis.

TablE (4): Simulation results of series No.1

\begin{tabular}{|c|c|c|c|c|c|c|c|}
\hline \multirow{2}{*}{$\begin{array}{l}\text { Test } \\
\text { No. }\end{array}$} & \multirow{2}{*}{$\boldsymbol{\beta}$} & \multirow[t]{2}{*}{$\mathrm{b}$} & \multicolumn{5}{|c|}{ Unreinforced Slope } \\
\hline & & & $\begin{array}{l}\text { Ordinary } \\
\text { Fellenius }\end{array}$ & $\begin{array}{c}\text { Bishop } \\
\text { Simplified }\end{array}$ & $\begin{array}{c}\text { Janbu } \\
\text { Corrected }\end{array}$ & Spencer & $\begin{array}{c}\text { MorgensternPri } \\
\text { ce }\end{array}$ \\
\hline $1-4$ & $30^{\circ}$ & $\begin{array}{l}1 B \\
2 B \\
3 B \\
4 B\end{array}$ & $\begin{array}{l}0.562 \\
0.655 \\
0.768 \\
0.864\end{array}$ & $\begin{array}{l}0.610 \\
0.752 \\
0.812 \\
0.926\end{array}$ & $\begin{array}{l}0.620 \\
0.722 \\
0.820 \\
0.898\end{array}$ & $\begin{array}{l}0.624 \\
0.758 \\
0.815 \\
0.940\end{array}$ & $\begin{array}{l}0.640 \\
0.761 \\
0.834 \\
0.971\end{array}$ \\
\hline $5-8$ & $35^{\circ}$ & $\begin{array}{l}1 B \\
2 B \\
3 B \\
4 B\end{array}$ & $\begin{array}{l}0.418 \\
0.484 \\
0.568 \\
0.660\end{array}$ & $\begin{array}{l}0.543 \\
0.582 \\
0.620 \\
0.761\end{array}$ & $\begin{array}{l}0.464 \\
0.546 \\
0.625 \\
0.728\end{array}$ & $\begin{array}{l}0.597 \\
0.614 \\
0.653 \\
0.767\end{array}$ & $\begin{array}{l}0.597 \\
0.612 \\
0.673 \\
0.779\end{array}$ \\
\hline $9-12$ & $40^{\circ}$ & $\begin{array}{l}1 B \\
2 B \\
3 B \\
4 B\end{array}$ & $\begin{array}{l}0.271 \\
0.360 \\
0.377 \\
0.507\end{array}$ & $\begin{array}{l}0.400 \\
0.453 \\
0.504 \\
0.590\end{array}$ & $\begin{array}{l}0.327 \\
0.414 \\
0.452 \\
0.563\end{array}$ & $\begin{array}{l}0.503 \\
0.516 \\
0.566 \\
0.656\end{array}$ & $\begin{array}{l}0.506 \\
0.551 \\
0.635 \\
0.655\end{array}$ \\
\hline
\end{tabular}




\subsection{Effect of length of geogrid layer}

To provide adequate length of the geogrid layer, two sets of tests were carried out on strip footing reinforced slope of $\beta=35^{\circ}$ with $N=4$ of SS30 geogrid of different $L_{r}=7 B$ and $10 B$ lengths. The test results of this series No.2 with different footing locations relative to slope edge $b / B=1,2$, 3 and 4 were presented in Table (5). Generally, it was noticed that the safety factor increases as $b / B$ increases for both lengths of geogrids considered. In addition, safety factors obtained with $L_{r}=10 B$ were higher than those obtained with $L_{r}=7 B$.
This behavior was mainly due to insufficient anchorage length of geogrid when $L_{r}=7 B$ which makes the mobilized lateral resistance comes from passive, interlocking and friction in the soil mass was smaller than transferred shear stresses. This in turn leads to pullout failure of geogrid layers with soil movement underneath the footing.

Figure (9) shows some results of series No.2 as critical slip surfaces and associated minimum safety factors of SS30 geogrid reinforced slope with $N=4$ of lengths $L_{r}=7 B$ and $10 B$ for $b / B=2$ by all analysis methods.

Table (5): Simulation results of series No.2

\begin{tabular}{|c|c|c|c|c|c|c|c|c|}
\hline \multirow{3}{*}{$\begin{array}{l}\text { Test } \\
\text { No. }\end{array}$} & \multirow[t]{3}{*}{$\boldsymbol{\beta}$} & \multirow[t]{3}{*}{$\mathrm{b}$} & \multirow[t]{3}{*}{$\mathrm{N}$} & \multicolumn{5}{|c|}{ Reinforced Slope with SS30 geogrid reinforcement } \\
\hline & & & & $\begin{array}{l}\text { Ordinary } \\
\text { Fellenius }\end{array}$ & $\begin{array}{c}\text { Bishop } \\
\text { Simplified }\end{array}$ & $\begin{array}{c}\text { Janbu } \\
\text { Corrected }\end{array}$ & Spencer & $\begin{array}{l}\text { Morgenstern } \\
\text { Price }\end{array}$ \\
\hline & & & & \multicolumn{5}{|c|}{ (a) Length of geogrid reinforcement $\left(L_{r}\right)=7 \mathbf{B}$} \\
\hline \multirow[t]{2}{*}{ 13-16 } & $35^{\circ}$ & $\begin{array}{l}1 B \\
2 B \\
3 B \\
4 B\end{array}$ & 4 & $\begin{array}{l}0.489 \\
0.584 \\
0.650 \\
0.681\end{array}$ & $\begin{array}{l}0.646 \\
0.687 \\
0.731 \\
0.761\end{array}$ & $\begin{array}{l}0.607 \\
0.658 \\
0.735 \\
0.749\end{array}$ & $\begin{array}{l}0.668 \\
0.709 \\
0.720 \\
0.777\end{array}$ & $\begin{array}{l}0.679 \\
0.739 \\
0.762 \\
0.804\end{array}$ \\
\hline & \multicolumn{8}{|c|}{ (b) Length of geogrid reinforcement $\left(L_{r}\right)=10 \mathrm{~B}$} \\
\hline $17-20$ & $35^{\circ}$ & $\begin{array}{l}1 B \\
2 B \\
3 B \\
4 B\end{array}$ & 4 & $\begin{array}{l}1.130 \\
1.207 \\
1.292 \\
1.498\end{array}$ & $\begin{array}{l}1.131 \\
1.209 \\
1.293 \\
1.506\end{array}$ & $\begin{array}{l}1.133 \\
1.209 \\
1.297 \\
1.581\end{array}$ & $\begin{array}{l}1.130 \\
1.205 \\
1.293 \\
1.521\end{array}$ & $\begin{array}{l}1.131 \\
1.205 \\
1.293 \\
1.519\end{array}$ \\
\hline
\end{tabular}

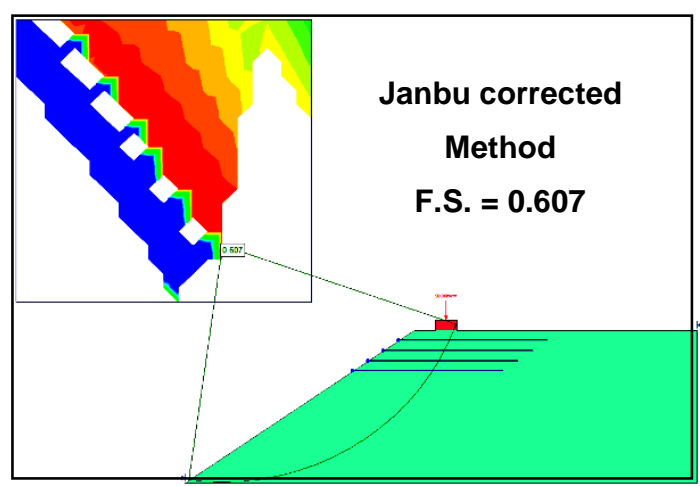

(a) $\beta=35^{\circ}, b / B=1, N=4$, and $L_{r}=7 B$.

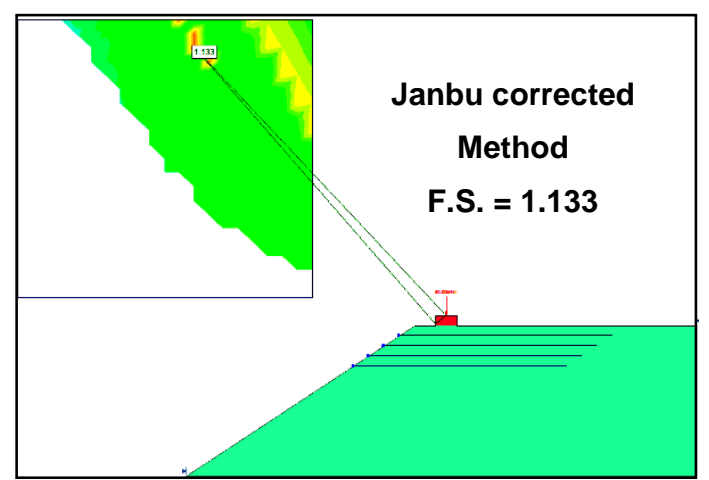

(b) $\beta=35^{\circ}, b / B=1, N=4$, and $L_{r}=10 B$.

Fig. (9): Minimum safety factors for some tests of series No.2. 

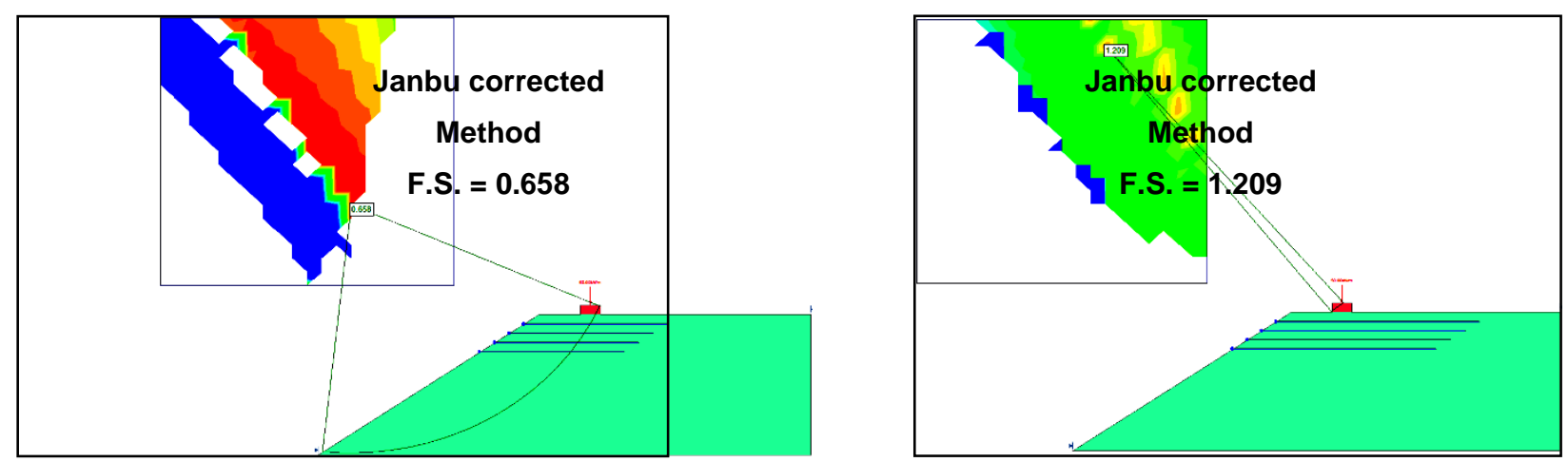

(c) $\beta=35^{\circ}, b / B=2, N=4$, and $L_{r}=7 B$.

(d) $\beta=35^{\circ}, b / B=2, N=4$, and $L_{r}=10 B$.

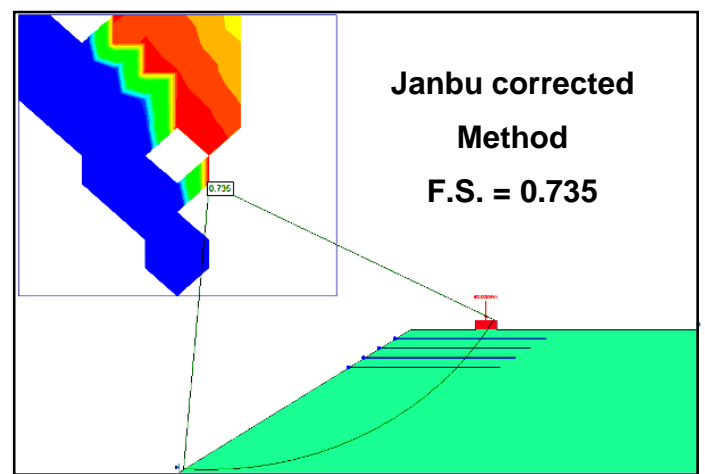

(e) $\beta=35^{\circ}, b / B=3, N=4$, and $L_{r}=7 B$.

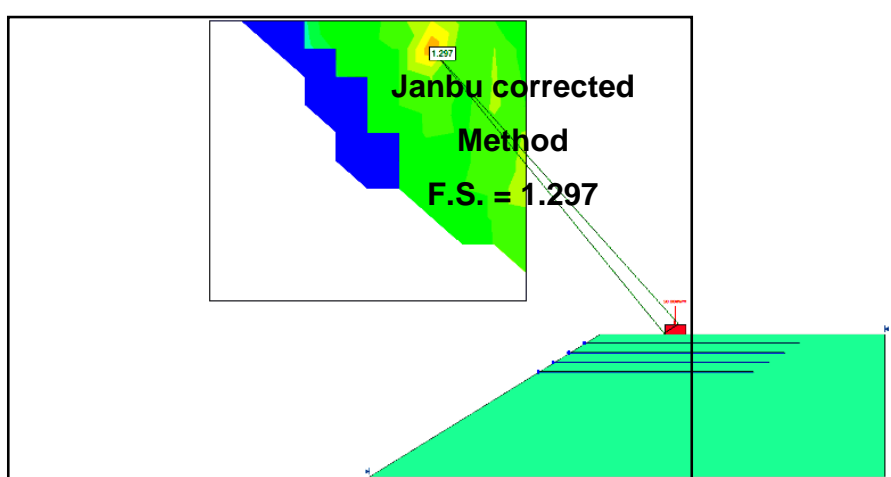

(f) $\beta=35^{\circ}, b / B=3, N=4$, and $L_{r}=10 B$.

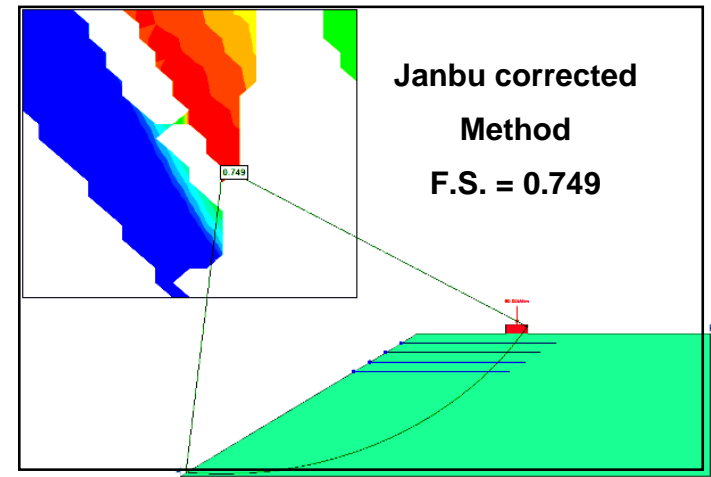

(g) $\beta=35^{\circ}, b / B=4, N=4$, and $L_{r}=7 B$.

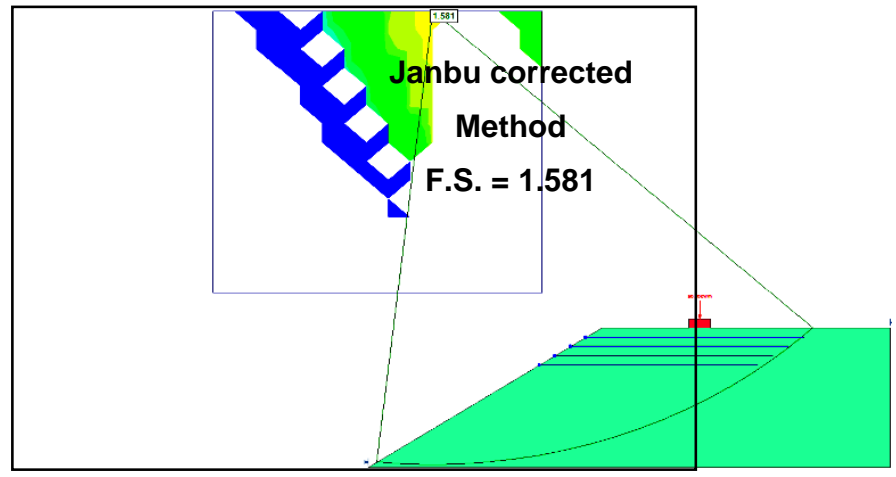

(h) $\beta=35^{\circ}, b / B=4, N=4$, and $L_{r}=10 B$.

Fig. (9): Cont'd.

\subsection{Effect of number of geogrid layers}

The test results of reinforced slope with $\mathrm{SS}_{30}$ geogrid were presented in Table (6). This table shows that for all footing locations from slope edge $b / B$ and all methods considered, the safety factor increases as the number of reinforcing layers $N$ increases. The increase in safety factor was evident due to the combined effects of geogrid and soil (sand) result from reinforcement mechanisms. In addition, the mobilized tensile stresses in geogrid reinforcements allows them to resist the built up shear stresses developed in the soil mass inside the loaded area. Then the stresses were transferred to the underlying layers of soil leading to longer and deeper failure zone, Alamshahi and Hataf (2009). The major benefit was obtained with $N=4$ when stress penetration reached $2 \mathrm{~B}$ below the footing, whereas with increasing $N$ to 5 layers no improvement was obtained. Some of this series No.3 test results were shown in Fig.(10). 
Table (6): Simulation results of series No.3

\begin{tabular}{|c|c|c|c|c|c|c|c|c|}
\hline \multirow{2}{*}{$\begin{array}{l}\text { Test } \\
\text { No. }\end{array}$} & \multirow[t]{2}{*}{$\bar{\beta}$} & \multirow[t]{2}{*}{$\mathrm{b}$} & \multirow[t]{2}{*}{$\mathrm{N}$} & \multicolumn{5}{|c|}{ Reinforced Slope with SS30 geogrid reinforcement } \\
\hline & & & & $\begin{array}{l}\text { Ordinary } \\
\text { Fellenius }\end{array}$ & $\begin{array}{c}\text { Bishop } \\
\text { Simplified }\end{array}$ & $\begin{array}{c}\text { Janbu } \\
\text { Corrected }\end{array}$ & Spencer & $\begin{array}{l}\text { Morgenstern } \\
\text { Price }\end{array}$ \\
\hline $21-40$ & $35^{\circ}$ & 1B & $\begin{array}{l}1 \\
2 \\
3 \\
4 \\
5\end{array}$ & $\begin{array}{l}0.569 \\
0.748 \\
0.940 \\
1.130 \\
1.130\end{array}$ & $\begin{array}{l}0.751 \\
0.980 \\
1.127 \\
1.131 \\
1.131\end{array}$ & $\begin{array}{l}0.671 \\
0.866 \\
1.076 \\
1.133 \\
1.133\end{array}$ & $\begin{array}{l}0.750 \\
0.951 \\
0.997 \\
1.130 \\
1.130\end{array}$ & $\begin{array}{l}0.814 \\
0.995 \\
1.130 \\
1.131 \\
1.131\end{array}$ \\
\hline & & $2 \mathrm{~B}$ & $\begin{array}{l}1 \\
2 \\
3 \\
4 \\
5\end{array}$ & $\begin{array}{l}0.434 \\
0.742 \\
1.007 \\
1.207 \\
1.208\end{array}$ & $\begin{array}{l}0.703 \\
0.970 \\
1.132 \\
1.209 \\
1.209\end{array}$ & $\begin{array}{l}0.533 \\
0.855 \\
1.085 \\
1.209 \\
1.212\end{array}$ & $\begin{array}{l}0.731 \\
0.831 \\
1.036 \\
1.205 \\
1.208\end{array}$ & $\begin{array}{l}0.893 \\
0.971 \\
1.136 \\
1.205 \\
1.208\end{array}$ \\
\hline & & 3B & $\begin{array}{l}1 \\
2 \\
3 \\
4 \\
5\end{array}$ & $\begin{array}{l}0.454 \\
0.587 \\
0.944 \\
1.292 \\
1.292\end{array}$ & $\begin{array}{l}0.725 \\
0.845 \\
1.094 \\
1.293 \\
1.293\end{array}$ & $\begin{array}{l}0.608 \\
0.667 \\
1.110 \\
1.297 \\
1.297\end{array}$ & $\begin{array}{l}0.768 \\
0.895 \\
1.026 \\
1.293 \\
1.293\end{array}$ & $\begin{array}{l}0.824 \\
0.963 \\
1.079 \\
1.293 \\
1.293\end{array}$ \\
\hline & & 4B & $\begin{array}{l}1 \\
2 \\
3 \\
4 \\
5\end{array}$ & $\begin{array}{l}0.637 \\
0.736 \\
0.827 \\
1.498 \\
1.482\end{array}$ & $\begin{array}{l}0.789 \\
0.923 \\
1.013 \\
1.506 \\
1.492\end{array}$ & $\begin{array}{l}0.711 \\
0.823 \\
0.912 \\
1.581 \\
1.572\end{array}$ & $\begin{array}{l}0.758 \\
0.852 \\
0.980 \\
1.521 \\
1.507\end{array}$ & $\begin{array}{l}0.848 \\
0.938 \\
1.016 \\
1.519 \\
1.511\end{array}$ \\
\hline
\end{tabular}
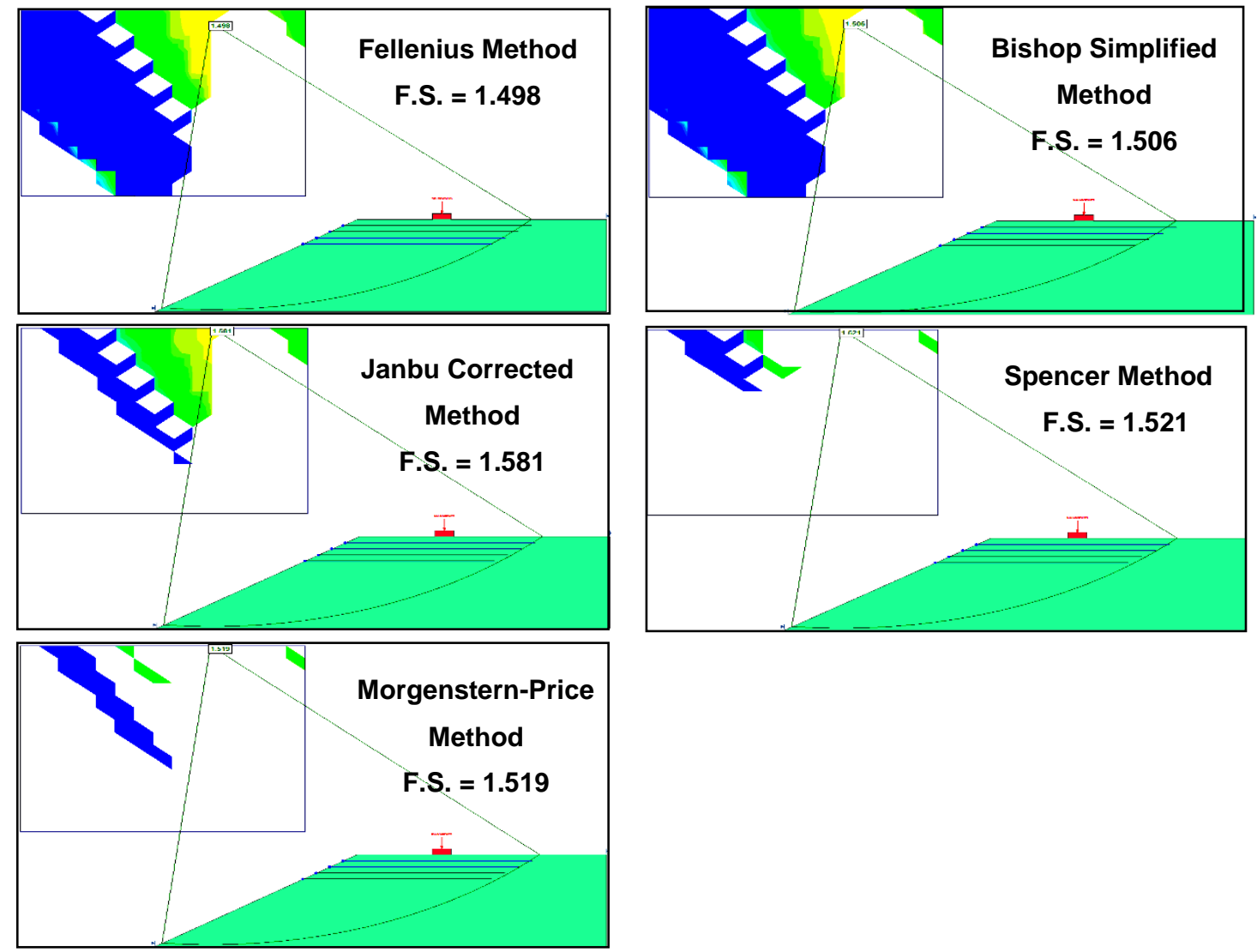

Fig (10): Minimum safety factors for some tests of series No.3 $\left(\beta=35^{\circ}, b / B=4, N=4, L_{r}=10 B\right.$ and $\left.\Delta v=0.5 B\right)$. 


\subsection{Effect of type of reinforcement}

Two different types of uniaxial and biaxial geogrids were used as reinforcement through two sets of tests with all other parameters were kept constant. The test results of this series No.4 were presented in Table (7). It can be seen that the slope reinforced by four layers of SS30 geogrid gives higher safety factors than that reinforced by TT045 geogrid for all footing locations from slope edge $b / B$ considered. This indicates that the reinforcement effect of SS30 biaxial geogrid is superior to that of TT045 uniaxial with highest safety factors obtained at $b / B=4$ and $N=4$. This result mainly was due to high pullout strength of SS30 geogrid and its properties such as; structure, thickness, aperture size, shape and rigidity, junction strength as well as grid modulus and stability.

Table (7): Simulation results of series No.4

\begin{tabular}{|c|c|c|c|c|c|c|c|c|}
\hline $\begin{array}{l}\text { Test } \\
\text { No. }\end{array}$ & $\boldsymbol{\beta}$ & $\mathrm{b}$ & $\mathrm{N}$ & $\begin{array}{l}\text { Ordinary } \\
\text { Fellenius }\end{array}$ & $\begin{array}{c}\text { Bishop } \\
\text { Simplified }\end{array}$ & $\begin{array}{c}\text { Janbu } \\
\text { Corrected }\end{array}$ & Spencer & $\begin{array}{l}\text { Morgenstern } \\
\text { Price }\end{array}$ \\
\hline & \multicolumn{8}{|c|}{ reinforcement } \\
\hline $41-44$ & $35^{\circ}$ & $\begin{array}{l}1 B \\
2 B \\
3 B \\
4 B\end{array}$ & 4 & $\begin{array}{l}0.786 \\
0.989 \\
1.041 \\
1.050\end{array}$ & $\begin{array}{l}0.953 \\
1.089 \\
1.126 \\
1.126\end{array}$ & $\begin{array}{l}1.083 \\
1.154 \\
1.162 \\
1.168\end{array}$ & $\begin{array}{l}0.884 \\
1.039 \\
1.077 \\
1.092\end{array}$ & $\begin{array}{l}1.075 \\
1.073 \\
1.100 \\
1.106\end{array}$ \\
\hline \multicolumn{9}{|c|}{ (b) Reinforced Slope with SS30 geogrid reinforcement } \\
\hline $45-48$ & $35^{\circ}$ & $\begin{array}{l}1 \mathrm{~B} \\
2 \mathrm{~B} \\
3 \mathrm{~B} \\
4 \mathrm{~B}\end{array}$ & 4 & $\begin{array}{l}1.130 \\
1.207 \\
1.292 \\
1.498\end{array}$ & $\begin{array}{l}1.131 \\
1.209 \\
1.293 \\
1.506\end{array}$ & $\begin{array}{l}1.133 \\
1.209 \\
1.297 \\
1.581\end{array}$ & $\begin{array}{l}1.130 \\
1.205 \\
1.293 \\
1.521\end{array}$ & $\begin{array}{l}1.131 \\
1.205 \\
1.293 \\
1.519\end{array}$ \\
\hline
\end{tabular}

\subsection{Effect of vertical spacing of the geogrid}

The tests of this series No.5 were conducted on model slope reinforced by 4 layers of SS30 geogrid with vertical spacing $\Delta v$ varied from $0.25 B$ to $1.50 B$ and the strip footing located at $b / B$ $=4$ from slope edge. The test results were presented in Table (8). As can be seen the optimum vertical spacing lies in the range of $0.50 B-0.75 B$ that gives maximum safety factor after which it decreases. Similar results were reported by Yoo (2001) and El-Sawwaf (2007) showing critical values of $\Delta v / B=0.70$ and 0.50 , respectively. Some of this series tests results were shown in Fig.

(11).

Table (8): Simulation results of series No.5

\begin{tabular}{|c|c|c|c|c|c|c|c|c|c|}
\hline \multirow{2}{*}{$\begin{array}{l}\text { Test } \\
\text { No. }\end{array}$} & \multirow[t]{2}{*}{$\boldsymbol{\beta}$} & \multirow[t]{2}{*}{$\mathrm{N}$} & \multirow[t]{2}{*}{$\mathrm{b}$} & \multirow[t]{2}{*}{$\Delta v$} & \multicolumn{5}{|c|}{ Reinforced Slope with SS30 geogrid reinforcement } \\
\hline & & & & & $\begin{array}{l}\text { Ordinary } \\
\text { Fellenius }\end{array}$ & $\begin{array}{c}\text { Bishop } \\
\text { Simplified }\end{array}$ & $\begin{array}{c}\text { Janbu } \\
\text { Corrected }\end{array}$ & Spencer & $\begin{array}{l}\text { Morgenstern } \\
\text { Price }\end{array}$ \\
\hline $49-53$ & $35^{\circ}$ & 4 & 4B & $\begin{array}{l}0.25 \mathrm{~B} \\
0.50 \mathrm{~B} \\
0.75 \mathrm{~B} \\
1.00 \mathrm{~B} \\
1.50 \mathrm{~B}\end{array}$ & $\begin{array}{l}1.472 \\
1.498 \\
1.482 \\
1.448 \\
1.405\end{array}$ & $\begin{array}{l}1.497 \\
1.506 \\
1.492 \\
1.482 \\
1.449\end{array}$ & $\begin{array}{l}1.562 \\
1.581 \\
1.582 \\
1.518 \\
1.470\end{array}$ & $\begin{array}{l}1.362 \\
1.521 \\
1.508 \\
1.519 \\
1.444\end{array}$ & $\begin{array}{l}1.498 \\
1.519 \\
1.515 \\
1.482 \\
1.448\end{array}$ \\
\hline
\end{tabular}



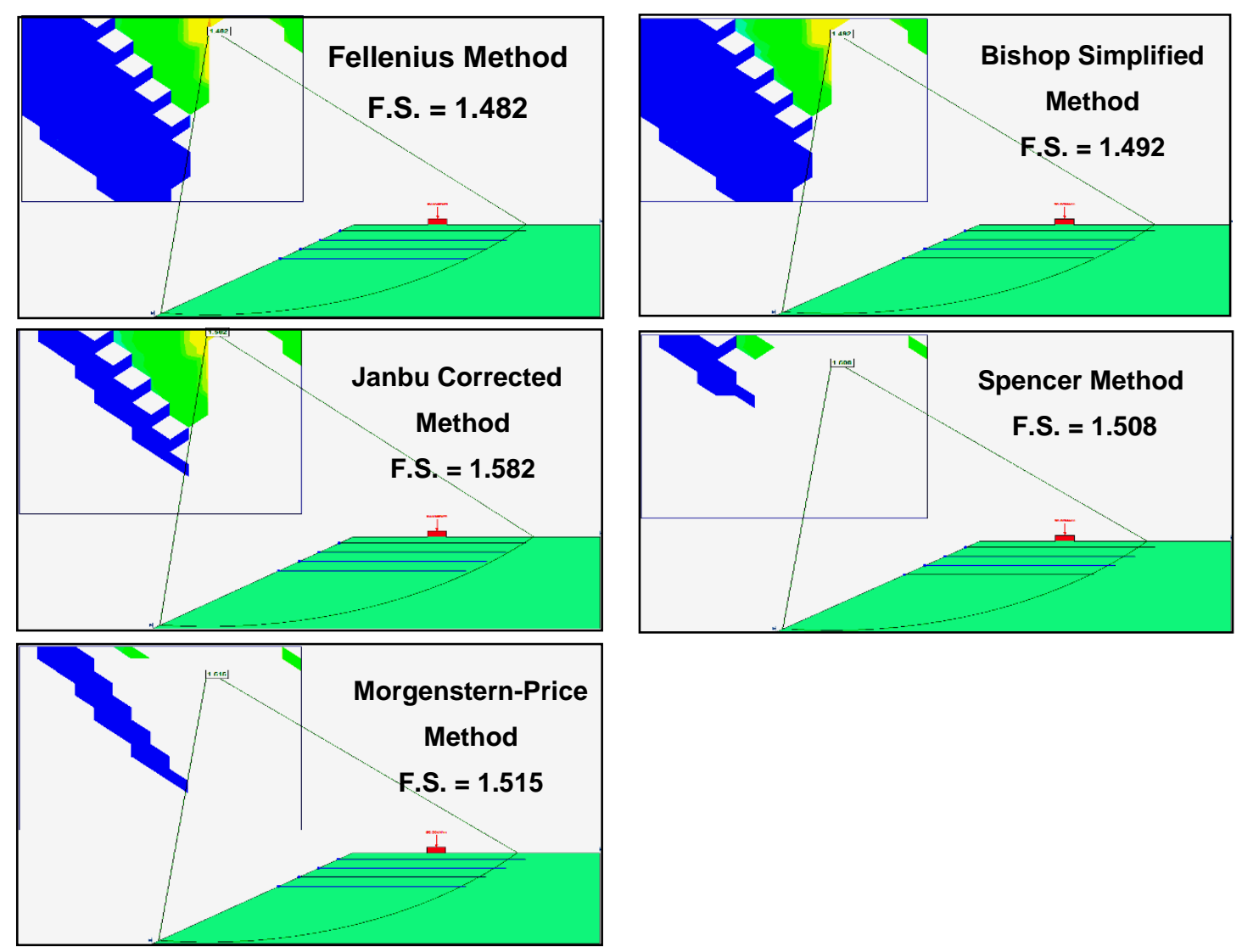

Fig, (11): Minimum safety factors for some tests of series No.5 $\left(\beta=35^{\circ}, b / B=4, N=4, L_{r}=10 B, \Delta v=0.75 B\right.$ and $\left.D_{f}=0\right)$.

\subsection{Effect of depth of footing}

The test results of this series No.6 were presented in Table (9). It can be seen that as the depth of footing increases, the safety factor increases as well. This behavior is mainly due to the presence of geogrid layers that prevent the soil particles from lateral movement toward the slope face and pushes them downward for deeper depth and hence spreads the footing load wider and deeper into the soil. This in turn leads to longer and deeper failure surface and greater bearing capacity or safety factor. Fig.(12) shows the critical slip surfaces and associated minimum safety factors for $\beta=35, b / B=4, N=4, \Delta v=$ $0.75 B$ and $D_{f}=B$ for all analysis methods.

Table (9): Simulation results of series No.6

\begin{tabular}{|c|c|c|c|c|c|c|c|c|c|}
\hline \multirow{2}{*}{$\begin{array}{l}\text { Test } \\
\text { No. }\end{array}$} & \multirow[t]{2}{*}{$\boldsymbol{\beta}$} & \multirow[t]{2}{*}{$\mathrm{b}$} & \multirow[t]{2}{*}{$\bar{N}$} & \multirow[t]{2}{*}{$\overline{D_{f}}$} & \multicolumn{5}{|c|}{ Reinforced Slope with SS30 geogrid reinforcement } \\
\hline & & & & & $\begin{array}{l}\text { Ordinary } \\
\text { Fellenius }\end{array}$ & $\begin{array}{c}\text { Bishop } \\
\text { Simplified }\end{array}$ & $\begin{array}{c}\text { Janbu } \\
\text { Corrected }\end{array}$ & Spencer & $\begin{array}{c}\text { Morgenstern } \\
\text { Price }\end{array}$ \\
\hline $54-55$ & $35^{\circ}$ & 4B & 4 & $\begin{array}{c}0 \\
0.5 \mathrm{~B} \\
1.0 \mathrm{~B}\end{array}$ & $\begin{array}{l}1.482 \\
1.513 \\
1.932\end{array}$ & $\begin{array}{l}1.492 \\
1.539 \\
1.826\end{array}$ & $\begin{array}{l}1.582 \\
1.613 \\
2.029\end{array}$ & $\begin{array}{l}1.508 \\
1.506 \\
2.246\end{array}$ & $\begin{array}{l}1.515 \\
1.520 \\
2.247\end{array}$ \\
\hline
\end{tabular}



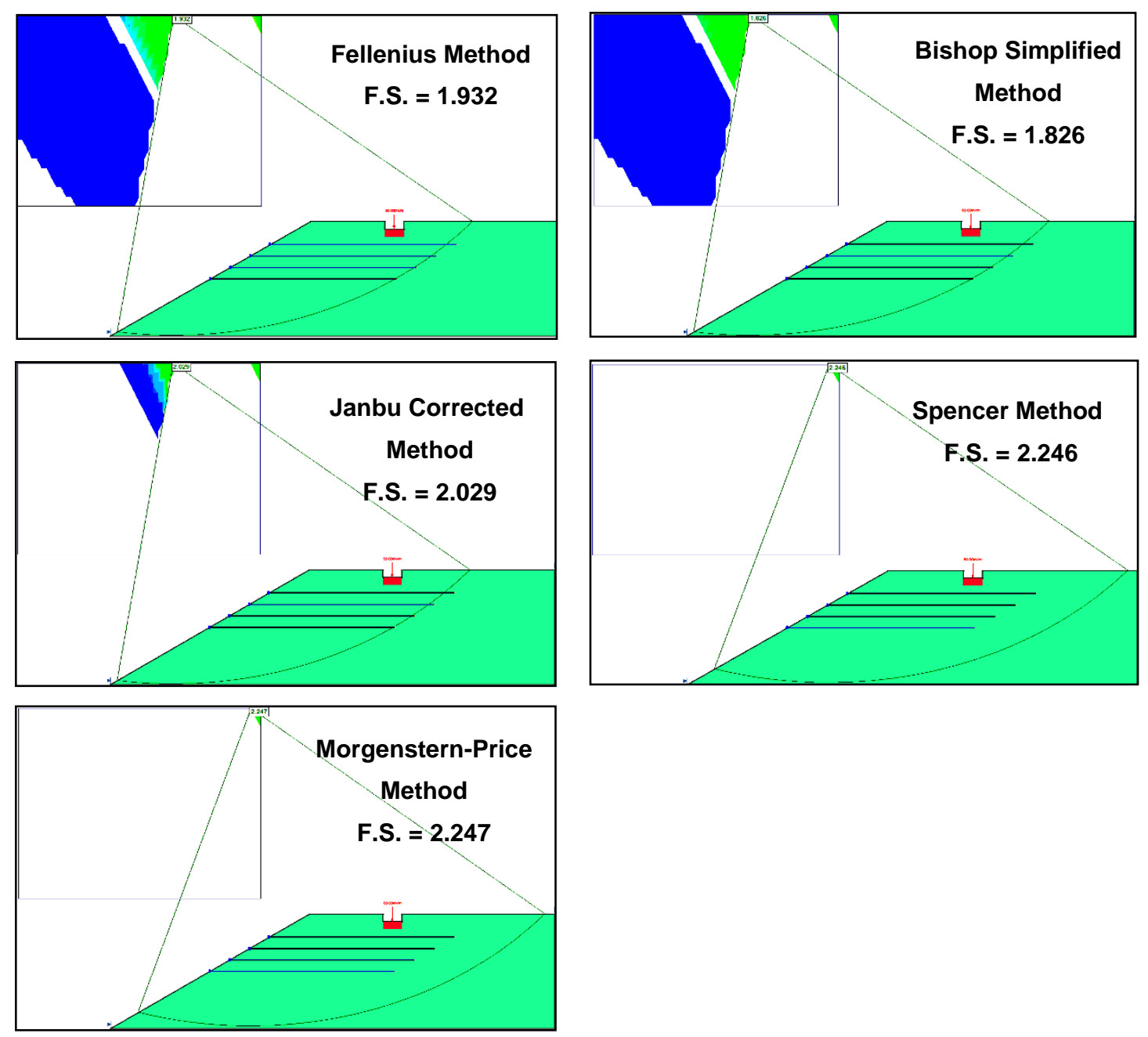

Fig. (12): Minimum safety factors for tests series No.6 $\left(\beta=35^{\circ}, b / B=4, N=4, L_{r}=10 B, \Delta v=0.75 B\right.$ and $\left.D_{f}=B\right)$.

\section{CONCLUSIONS}

Based on results of this study, the following conclusions are drawn:

1. Slide v6 software computer program is efficient to study the comparative cost of various options of any given slope stability reinforced or unreinforced problem both for research and design purposes.

2. Finding the safety factor of sloped embankment by limit equilibrium methods requires an algorithm such as grid search to find the critical slip circle using rigorous procedures which satisfy both force and moment equilibriums such as Spencer's or Morgenstern and Price methods.

3. For any slope angle considered, the safety factor increases as the footing distance away from edge of slop $b / B$ increases.

4. The inclusion of geogrid reinforcement would increase slope stability. The major benefit was obtained when the number of reinforcing layers $N$ $=4$.

5. The adequate length of geogird reinforcement $L_{r}$ for anchorage purposes does not less than 10 times the width of footing. Hence, longer geogrid layers mobilize greater resistance up to maximum pullout capacity of them after which the slope system fails.

6. SS30 geogrid is more effective for reinforcing slopes than TT045 geogrid due to its higher pullout strength and other properties such as structure, shape, rib profile and grid junction tensile strength that affecting soil/grid interlock and increasing the safety of reinforced slopes.

7. The optimum depth of the upper most layer of reinforcement was $u=0.5 B$ where $(B=$ width of footing) whereas the optimum vertical spacing of the reinforcement layers $\Delta v$ lies in the range of $0.50 B-0.75 B$. 


\section{REFERENCES}

- Alamshahi, S., and Hataf, N. (2009), "Bearing capacity of strip footings on sand slopes reinforced with geogrid and grid anchor", Geotextiles and Geomembranes, 27, pp.217226.

- Choudhary, A.K., Jha, J.N. and Gill, K.S. (2010),"Laboratory investigation of bearing capacity behavior of strip footing on reinforced flyash slope", Geotextiles and Geomembranes, 28, pp.393-402

- Elias V. and Christopher B. (2001), "Mechanically stabilized earth walls and reinforced soil slopes design and construction guidelines", National Highway Institute, Department of Transportation FHWA, Washington, D.C., USA.

- El-Sawwaf, M. (2007),"Behavior of strip footing on geogrid-reinforced sand over a soft clay slope", Geotextiles and Geomembranes, 25(1), pp.5060.

- Gill, K.S., Kaur, A., Choudary, A.K., and Jha, J.N. (2011), "Numerical study of footing on single layer reinforced slope", Proceedings of Indian Geotechnical Conference December 15-17, 2011, Kochi (Paper No. N-120), pp.839-842.

- Keskin M. S., and Laman M. (2014), "Experimental and numerical studies of strip footings on geogrid- reinforced sand slope, Arabian Journal for Science and Engineering, Vol. 39, pp.16071619.

- Laman, M. and Yildiz, A. (2003), "Model studies of ring foundations on geogrid-reinforced sand",
International Journal of Geosynthetics, Vol. 10, No. 5, pp. 142-152.

- Lee, K.M., and Manjunath, V.R. (2000), "Experimental and numerical studies of geosynthetic reinforced sand slopes loaded with footing", Canadian Geotechnical Journal, Vol.37, pp.828-842.

- [9] Mirafi (1998), "Geosynthetics for soil reinforcement", Reinforced soil engineering, Ten Cate Nicolon, pp.1-30.

- Shi, Denda and Wang, Fei (2013), "Pullout test studies on the interface characteristics between geogrids and soils", Vol.18, pp.5405-5417, EJGE.

- Strata Systems (2010),"Reinforced soil slopes and embankments", Strata Global GeoSolutions. http://www.geogrid.com/ images/ stories/ downloads/ slopemanual. pdf__ (accessed February 2015).

- Tenax, Technical Application Document No. DVT0001 (2001), ITC Resolution No. 058/11. CE market. Geogrids TENAX "TT SAMP 045, 060, 090, 120, 160', Italy.

- Tensar International (2003), "The properties and performance of tensar biaxial geogrids". Tensar International Limited. http://www.tensarinternational.com (accessed Febraury 2015).

- Yoo, C. (2001), "Laboratory investigation of bearing capacity behavior of strip footing on geogrid reinforced sand slope", Geotextiles and Geomembranes, 19 (5), pp.279- 298. 
شيكرنا جيّكيربووما سهرنشيفهى خيزى جه كدار لبن باركرنا بنياتهكن كنفى

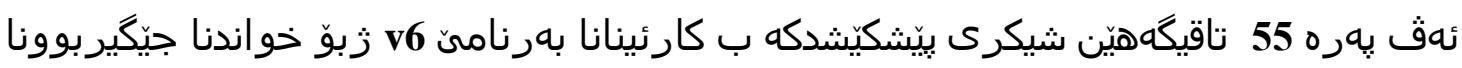

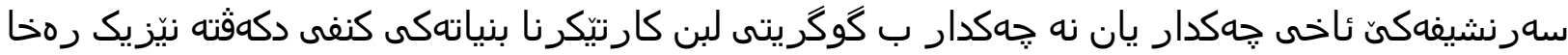

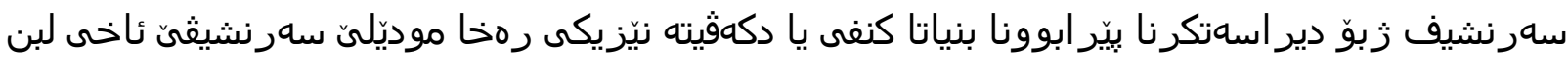

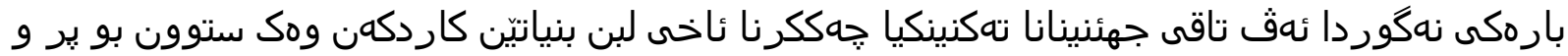

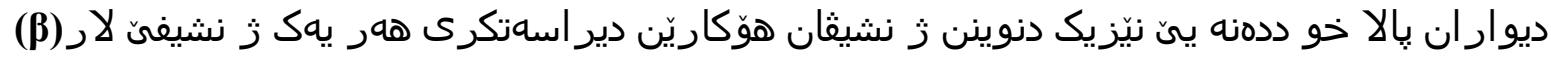

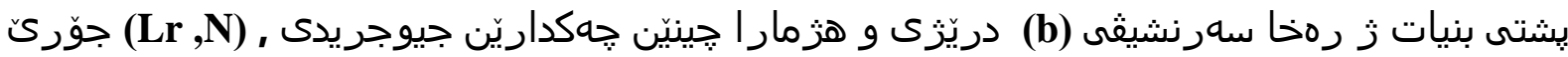

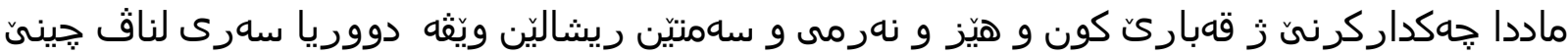

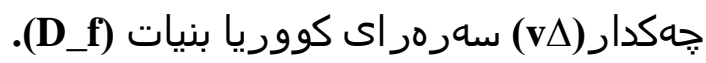

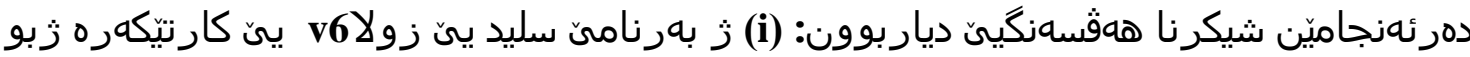

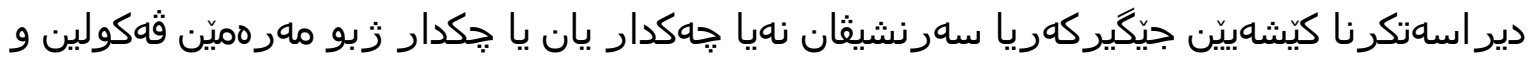

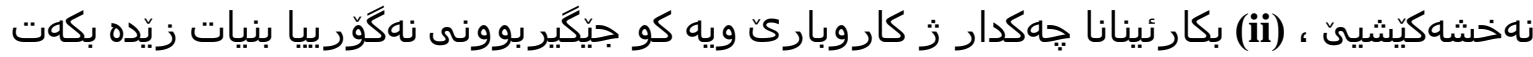

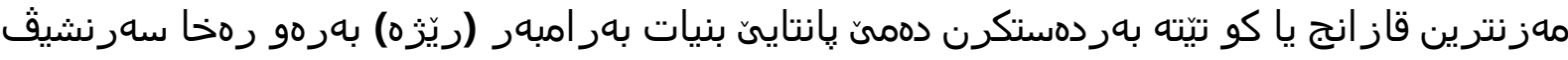

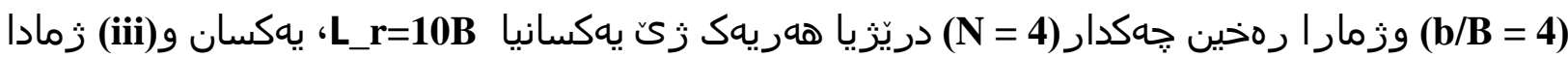

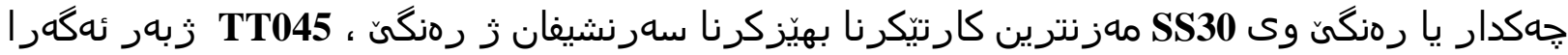

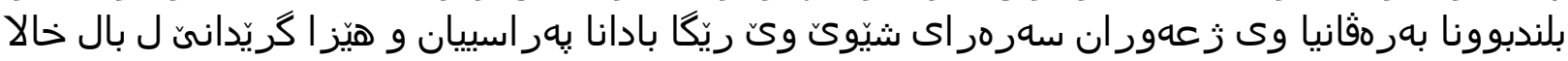

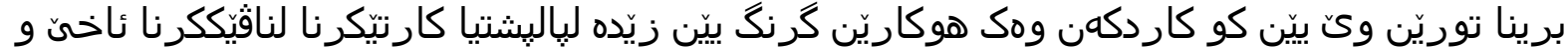

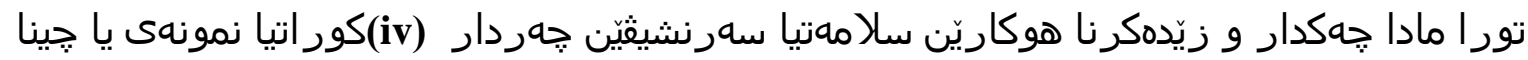

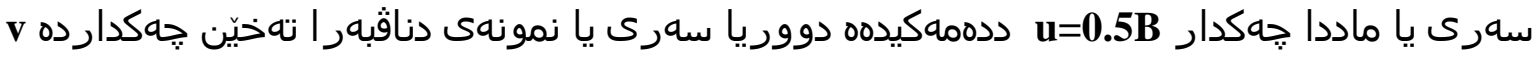

دكهيته خاليّن . B-0.75B0.5

تحليل إستقرارية مذحدر رملي مسلح تحت تحميل أساس شريطي 
تقدم هذه الورقة 55 إختبارتحليل باستخدام برنامج الشريحة v6 لدراسة إستقرارية منحدر ترابي الإبي

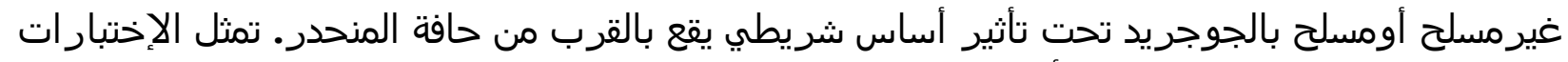

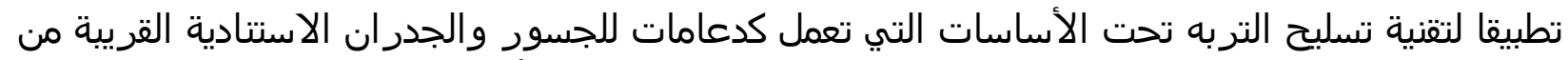

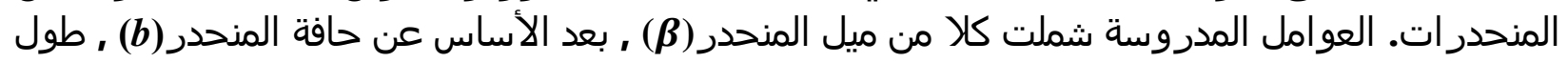

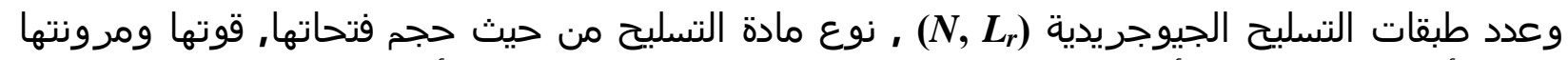

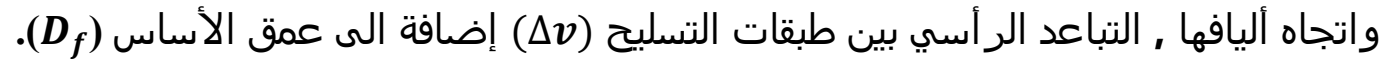

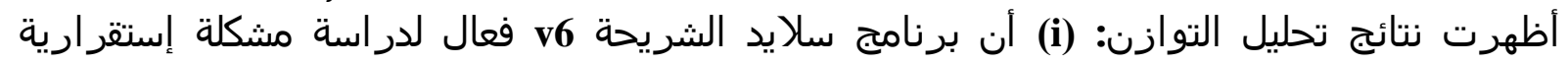

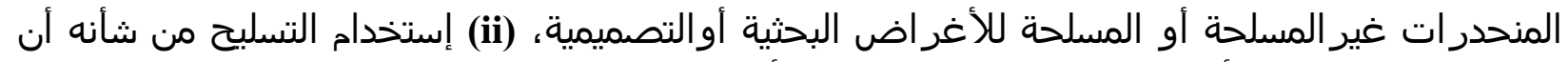

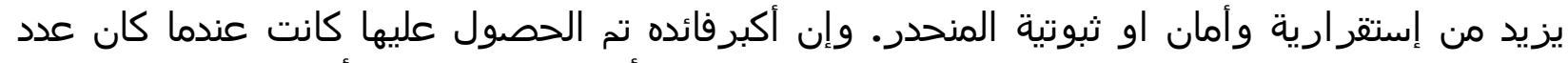

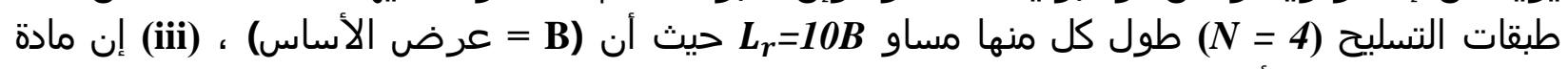

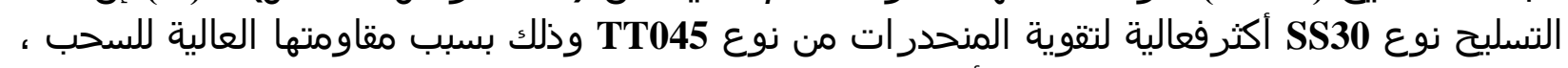

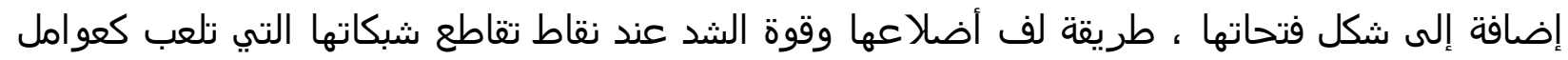

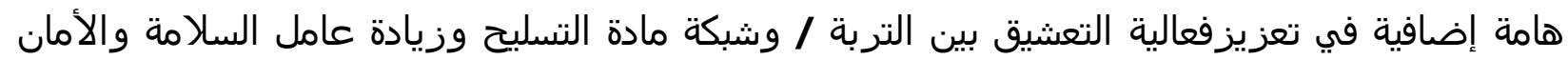
اللمنحدرات المسلحة، (iv) إن العمق الأمثل للطبقة العبية العليا لمادة التسليج هو الرأسي الأمثل بين طبقات التسليح $\Delta v$ يقع في نطاق 